\title{
Strates
}

STRATES Matériaux pour la recherche en sciences sociales

$4 \mid 1989$

Dossier : images réfléchies. Paroles d'un paysan révolutionnaire

\section{L'espace et le temps en Camargue de Bernard Picon : une relecture}

\section{Françoise Plet}

\section{(2) OpenEdition}

Journals

Édition électronique

URL : http://journals.openedition.org/strates/4872

DOI : $10.4000 /$ strates.4872

ISSN : $1777-5442$

Éditeur

Laboratoire Ladyss

Édition imprimée

Date de publication : 31 décembre 1989

ISSN : 0768-8067

Référence électronique

Françoise Plet, "L'espace et le temps en Camargue de Bernard Picon : une relecture », Strates [En ligne], 4 | 1989, mis en ligne le 19 mai 2008, consulté le 08 septembre 2020. URL : http:// journals.openedition.org/strates/4872 ; DOI : https://doi.org/10.4000/strates.4872

Ce document a été généré automatiquement le 8 septembre 2020.

Tous droits réservés 


\title{
L'espace et le temps en Camargue de Bernard Picon : une relecture ${ }^{1}$
}

\author{
Françoise Plet
}

1 J'avais pris grand plaisir en 1978 à découvrir la première édition de ce livre paru sous la belle couverture kraft qui était alors la signature de l'éditeur. Je l'ai largement utilisé dans mon travail d'enseignement, que les thèmes en soient l'apprentissage de la lecture de paysages et de cartes, les espaces ruraux ou les questions d'aménagement. Il représente, dans sa nouvelle édition comme hier, le guide de qui veut aller en Camargue, et un incomparable outil d'intelligibilité pour qui en revient.

2 Le projet - le décryptage des relations entre société, activités et territoire - est exposé dans le chapitre introductif sous l'hypothèse que "la Camargue ne serait... point un découpage arbitraire de l'espace, mais un lieu où tous les rapports... entre les différents acteurs passent par un dénominateur commun - la gestion de l'espace - et se nouent autour de cet unique enjeu ». L'objectif est tenu, avec une maîtrise des éléments qui construisent l'organisation et les dynamiques spatiales de nature à rendre jaloux maint géographe. Il est possible de constater dix ans plus tard que l'analyse permettait aussi d'anticiper, ou du moins de ne pas être surpris de telle information ${ }^{2}$ relatant l'achat de 200 hectares en Camargue et l'implantation du restaurant «La datcha du guardian»(!) par Francine Gomez, ex-PDG de Waterman, ou de la reprise récente de la riziculture, en voie de disparition en 1978 (14 000 ha en 1988). La perspective adoptée n'accorde peutêtre cependant pas suffisamment d'attention, pour la période actuelle, à toutes les relations entre les activités camarguaises. L'élevage du cheval notamment est-il totalement indépendant de la grande agriculture? N'existe-t-il pas aujourd'hui une synergie familiale dans l'utilisation productive des sols au sein de laquelle papa produit du riz et du blé dur tandis que le fils élève et dresse des animaux de race Camargue?

3 L'édition parue à l'extrême fin 1988 met à jour les évolutions constatées dans la décennie. Mais elle n'est pas que cela. Bien que l'économie d'ensemble du livre demeure, qu'une partie du texte soit conservée, il s'agit aussi d'un autre livre, par le choix des titres de chapitres comme par la sélection et les remodelages du corps de l'ouvrage, qui témoignent de l'évolution intellectuelle de l'auteur tout autant que de 
raffinement de son rapport à la Camargue. La première version portait en sous-titre "Essai d'écologie sociale». La bibliographie comme les annexes témoignaient d'une découverte récente et assez enthousiaste des relations nature-société et de la géographie. Le ton des intitulés et une partie du texte se situaient dans la ligne " engagée » adoptée par nombre de chercheurs des années soixante-dix, laquelle visait à montrer et démontrer le rôle des "idéologies dominantes" dans l'évolution des structures sociales, des rapports à la nature, et l'exploitation de la nature. La bibliographie de 1988 est beaucoup plus composée de travaux de sociologues, et d'études camarguaises. Les titres, lorsqu'ils ont été transformés, sont à la fois plus neutres et «modernisés ». Dans le texte, nombre de références aux rapports de forces, aux acteurs, au rôle des capitaux, des investissements, des rapports marchands, sont supprimés, surtout s'il s'agissait de considérations d'ordre général, au profit d'un recentrage sur l'objet "Camargue». Au contraire, les perspectives de l'ordre de l'imaginaire, du rituel ou du symbolique prennent plus de force. Les cartes et croquis apparaissent moins étroitement associés au texte décrivant le territoire "Camargue ", ne serait-ce qu'au niveau de la mise en pages pour ceux d'entre eux qui ont été conservés. Des changements subtils témoignent indirectement des évolutions sociales, ou idéologies, de la décennie (" les derniers gardians salariés» de 1978 se transforment en "certains cavaliers" en 1988; "compétition pour l'affectation des sols" se change en "compétition pour la gestion hydraulique»; le «capitalisme» de 78 devient «la bourgeoisie marchande marseillaise et régionale" en 1988...). De nouveaux développements apparaissent autour de l'idée de "paysage ». A une attitude réservée à l'égard de la Réserve Naturelle succède semble-t-il une attitude plutôt favorable. La question du jeu des décisions politiques et administratives, très présente en 1978, est minorisée. Les précisions factuelles gagnent sur les interprétations. L'«objectif» transcende l'idéologie.

4 J'ai quant à moi un peu regret de la disparition d'une certaine vigueur contestataire. J'ai aussi le regret que sa prise de distance vis-à-vis d'enthousiasmes de néophyte en matière de géographie et d'écologie n'ait pas conduit Bernard PICON à reconstruire totalement son ouvrage autour de son discours fondamental (la deuxième partie: «L'espace objet » dans le texte de 1988), en y intégrant plus étroitement la chronologie, convenue et seulement explicite a posteriori, qui fait l'objet des soixante-dix premières pages. Celle-ci continue de poser l'histoire préindustrielle comme prolégomène à la structuration socio-spatiale étudiée, ce qui m'apparaît relever d'une vision encore faiblement élaborée du rôle des éléments du passé et des échelles du temps dans les constructions spatiales observables.

5 Mais ces réserves ne réduisent en rien l'intérêt de cette approche de la Camargue, ni l'apport intellectuel et pédagogique du livre. Lisez donc les deux versions ! 


\section{NOTES}

1. Bernard PICON, 1988, L'espace et le temps en Camargue, Arles, Actes Sud, 231 p., cartes et figures.

2. A pour Affaires Economiques, $n^{\circ} 2$, octobre 1989

\section{AUTEUR}

\section{FRANÇOISE PLET}

Maître de conférences à l'université Paris-VIII, elle est spécialiste du domaine agroalimentaire. 